\title{
Estudo transversal do autorrelato de bruxismo e sua associação com estresse e ansiedade
}

\author{
Cross-sectional study about self-reported bruxism and its association with \\ stress and anxiety
}

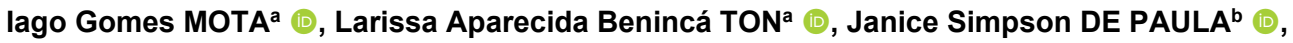 \\ Ana Paula Varela Brown MARTINS ${ }^{a^{*}}$ (1) \\ aUFJF - Universidade Federal de Juiz de Fora, Governador Valadares, MG, Brasil \\ 'UFMG - Universidade Federal Minas Gerais, Belo Horizonte, MG, Brasil
}

\begin{abstract}
Como citar: Mota IG, Ton LAB, De Paula JS, Martins APVB. Estudo transversal do autorrelato de bruxismo e sua associação com estresse e ansiedade. Rev Odontol UNESP. 2021;50:e20200003. https://doi.org/
\end{abstract}

\begin{abstract}
Resumo
Introdução: 0 bruxismo é uma condição prevalente e pode ser destrutivo à cavidade bucal, sendo amplamente abordado na Odontologia, especialmente por sua etiologia multifatorial e prevalência controversa. Objetivo: Este estudo teve o objetivo de investigar a prevalência do autorrelato de bruxismo entre universitários e correlacionar a parafunção com a ansiedade e o estresse autopercebidos. Material e método: Utilizaram-se o autorrelato de bruxismo, Inventário de Ansiedade Traço-Estado (IDATE) e Inventário de Sintomas de Estresse de LIPP (LIPP) para avaliar a prevalência de bruxismo, ansiedade e estresse, respectivamente. Setecentos e quatorze estudantes de todos os cursos da universidade foram entrevistados, com idade entre 18 e 45 anos. Análises estatísticas descritivas foram realizadas por cálculos de frequências (absolutas e relativas), bem como medidas de tendência central e dispersão (média e desvio padrão). Quanto às análises estatísticas inferenciais, o teste qui-quadrado ou exato de Fisher foi utilizado na comparação de proporções e o teste $t$ de Student ou ANOVA, como critério para comparar médias, todos com nível de significância de 5\%. Resultado: A prevalência de bruxismo foi 46,92\%; houve diferença significante da proporão do bruxismo com a ansiedade estado $(p=0,00)$ e traço $(p \leq 0,0001)$ de personalidade. Conclusão: Conclui-se que, apesar de o bruxismo não ter prevalecido entre estudantes, um elevado percentual de estudantes afirmou realizar essa atividade repetitiva e a proporção de bruxistas com ansiedade foi relevante, evidenciando a importância desse fator psicológico na fisiopatologia da parafunção.
\end{abstract}

Descritores: Bruxismo; ansiedade; estresse psicológico.

\begin{abstract}
Introduction: Bruxism is a prevalent condition and can be destructive to the oral cavity, being widely addressed in dentistry, especially due to its multifactorial etiology and controversial prevalence. Objective: This study aimed to investigate the prevalence of self-reported bruxism among university students and to correlate it with self-perceived anxiety and stress. Material and method: Self-reported Bruxism, TraitState Anxiety Inventory (STAI) and LIPP Stress Symptoms Inventory (LIPP) were used to assess the prevalence of bruxism, anxiety and stress, respectively. 714 students of all courses of a university were interviewed, they were between 18 and 45 years. Descriptive statistical analyzes performed by calculations of frequencies (absolute and relative), as well as measures of central tendency and dispersion (mean and standard deviation). About inferential statistical analyzes, the Chi-square or Fisher's exact test was used to compare proportions and the Student $t$ test or ANOVA was a criterion for comparing means, all with a 5\% significance level. Result: The prevalence for bruxism was $46.92 \%$; there was a significant difference in the proportion of bruxism with state anxiety $(\mathrm{p}=0.00)$ and personality trait $(\mathrm{p}=<0.0001)$. Conclusion: It is concluded that although bruxism did not prevail among students, a high percentage of students stated to perform this repetitive activity and the proportion of bruxists with anxiety was relevant, showing the importance of this psychological factor in the pathophysiology of parafunction.
\end{abstract}

Descriptors: Bruxism; anxiety; psychological stress. 


\section{INTRODUÇÃO}

As interpretações atuais do bruxismo sugerem que pode ser considerado uma condição que pode se tornar um fator de risco para algumas consequências clínicas deletérias ${ }^{1}$. Na definição mais recente, foi descrito como uma atividade repetitiva dos músculos mastigatórios caracterizada por apertar ou ranger os dentes e/ou manter rígida ou mover vigorosamente a mandíbula. A atividade pode ocorrer de formas distintas, de acordo com o ciclo circadiano, classificada, assim, em bruxismo do sono (BS) e bruxismo em vigília (BV)².

0 bruxismo apresenta intervalos de prevalência de $8 \%$ a 31\%, sem distinção entre em vigília ou do sono, $22 \%$ a $31 \%$ para BV e $13 \% \pm 3 \%$ para BS em adultos ${ }^{3}$. Sua distribuição é indiferente quanto ao gênero, porém, em relação à faixa etária, são encontradas altas prevalências em crianças e adolescentes (por exemplo, 3,5\% a 40\% para BS), tendendo a diminuir com o aumento da idade ${ }^{1}$.

O bruxismo pode desenvolver alguns problemas clínicos, mais frequentemente estando associado a problemas mecânicos (afrouxamento de parafusos de prótese sobre implante, fratura de cerâmica, de pilar ou estrutura, fraturas ou falhas nas restaurações dentárias), embora complicações biológicas possam ocorrer (por exemplo, desgaste dos dentes, comprometimento

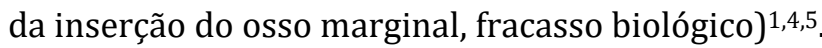

A literatura é controversa sobre a relação entre bruxismo e dor, sendo as mais citadas dor nos músculos mastigatórios e/ou nas articulações temporomandibulares, dor de cabeça ${ }^{4}$. Sugere-se que BS e BV podem ter etiologia diferente e as atividades de apertar e ranger são fenômenos motores diferentes com consequências potencialmente diversas em termos de fadiga muscular $\mathrm{e}$ estresse articular para explicar tal controvérsia ${ }^{1}$.

A influência da oclusão dentária sobre bruxismo não é mais aceita e, atualmente, é considerado de etiologia multifatorial, incluindo fatores biológicos, neurológicos (por exemplo, neuroquímicos, como dopamina e outros neurotransmissores, genética, despertar do sono), psicológicos (por exemplo, sensibilidade ao estresse, traços de personalidade, ansiedade) e exógenos (por exemplo, tabagismo, álcool, cafeína, certos medicamentos, como inibidores seletivos da recaptação de serotonina, drogas ilícitas) ${ }^{1,6}$.

Características de personalidade, como traços de ansiedade e sensibilidade ao estresse, são os principais fatores psicológicos associados ao bruxismo, especialmente $\mathrm{BV}$, independentemente da faixa etária ${ }^{7-9}$. 0 mecanismo fisiopatológico proposto é que indivíduos com altos níveis de sensibilidade a estresse, ansiedade, neuroticismo e traços de responsabilidade tendem a liberar tensão emocional, envolvendo atividades de BS e/ ou BV7. Logo, traços de personalidade e sensibilidade ao estresse foram identificados como fatores de risco entre adultos e adolescentes ${ }^{1,9,10}$. Assim, estudos baseados no autorrelato do bruxismo forneceram sua associação com alguns traços de personalidade, fortalecendo a opinião generalizada da existência de uma relação bruxismo-fatores psicossociais.

Portanto, o objetivo do estudo foi investigar a prevalência de possível bruxismo em adultos usando abordagem não instrumental e avaliar a associação com ansiedade e estresse psicológico. As hipóteses desse estudo foram: 1) elevada prevalência do autorrelato do bruxismo, ansiedade e estresse entre universitários; 2) autorrelato do bruxismo significantemente correlacionado com estresse e ansiedade.

\section{MATERIAL E MÉTODO}

Esta pesquisa caracterizou-se por ser um estudo de prevalência entre alunos da Universidade Federal de Juiz de Fora, campus Governador Valadares (UFJF-GV), aprovado pelo comitê de ética (no 1.878.567). 
O número total de universitários que participaram da pesquisa foi de 714 (482 mulheres), saudáveis, com idade variando de 18 a 40 anos (média de idade de 21,5 anos; desvio padrão \pm 4,53 anos). Foram recrutados alunos regularmente matriculados nos diversos cursos de graduação da UFJF-GV, representando $33,8 \%$ do total de alunos. 0 estudo foi realizado de acordo com as diretrizes da Declaração de Helsinque, completamente anônimo, com todos os indivíduos tendo assinado o termo de consentimento livre e esclarecido (TCLE) e podendo livremente se retirar do estudo a qualquer momento. Além do TCLE, todos os voluntários responderam aos questionários.

Para participar da pesquisa, os voluntários precisariam ter idade de 18 a 40 anos, capacidade de responder às perguntas e estar regularmente matriculados em um dos cursos da UFJF-GV. Os critérios de exclusão foram história de desordens neurológica, mental ou do sono, presença de doenças sistêmicas que pudessem confundir os resultados (discinesias oromandibular ea orofacial), traumas de face e uso contínuo de medicamentos (analgésicos, anti-inflamatórios, antidepressivos, anticonvulsivantes).

A coleta de dados dos alunos foi realizada imediatamente após o período de recesso de final de ano, com duração total de sete dias de coleta. A aplicação dos questionários foi realizada em diferentes locais de funcionamento da UFJF/GV, durante os horários das atividades acadêmicas. Antecedendo a aplicação dos questionários, cada voluntário recebeu uma carta de informação aos voluntários da pesquisa, que continha a finalidade da pesquisa e dos dados recolhidos e sua utilização.

Para obtenção do diagnóstico de ansiedade, estresse e bruxismo, foram utilizados o autorrelato de bruxismo, o Inventário de Ansiedade Traço-Estado (IDATE) e o Inventário de Sintomas de Estresse de LIPP (ISSL).

0 autorrelato de bruxismo foi investigado com o Índice Anamnésico de Fonseca*, um instrumento destinado a avaliar a prevalência e a gravidade dos distúrbios temporomandibulares. No item 8, questionava: "Já observou se tem o hábito de apertar e/ou ranger os dentes durante o dia ou a noite?", de modo que o aluno poderia assinalar uma das três seguintes respostas: "sim", "não" ou "às vezes". Os alunos que responderam "sim" e "às vezes" foram considerados bruxistas.

Para avaliação do nível de ansiedade, foi utilizado o IDATE, questionário de autorrelato. Constituído por duas escalas tipo Likert, a primeira relativa ao estado de ansiedade (ansiedadeestado, estado emocional momentâneo) e a segunda, ao traço de ansiedade (ansiedade-traço, considera a personalidade do indivíduo, predisposição a reagir com ansiedade em situações estressantes). Cada avaliação é constituída de 20 perguntas: a primeira avalia como o voluntário se sente em um determinado momento no tempo, enquanto a segundo questiona como geralmente se sente durante a vida. A classificação é feita de acordo com a soma dos pontos das respostas assinaladas: entre 20 e 29 pontos, não há ansiedade; entre 30 e 37, ansiedade baixa; entre 38 e 44, ansiedade moderada; entre 45 e 80, ansiedade grave. Para esse estudo, considerouse com ou sem ansiedade ${ }^{11}$.

O LIPP qualifica e quantifica os sintomas de estresse físicos e psíquicos e categoriza-os nas seguintes fases: alerta, resistência e exaustão. Esse questionário é formado por três quadros que contêm sintomas físicos e psíquicos de cada fase do estresse, sendo o número de sintomas físicos maior que o dos psicológicos, variando de fase para fase, contabilizando no total 34 itens de natureza somática e 19 de natureza psicológica ${ }^{12}$.

Para esse estudo, utilizou-se apenas o resultado da avaliação de estresse obtida nas últimas 24 horas prévias à aplicação do questionário. 0 diagnóstico é obtido do somatório dos itens marcados no questionário, em que cada resposta marcada é contabilizada como um ponto. No

* Fonseca DM. Disfunção craniomandibular (DCM): diagnóstico pela anamnese [Dissertação de Mestrado]. Bauru: Faculdade de Odontologia de Bauru da USP, 1992. 
final do primeiro quadro do questionário (que mede o nível de estresse das últimas 24 horas), se o número de respostas marcadas for igual ou superior a 9, o voluntário será classificado como um indivíduo que apresenta estresse.

Os dados obtidos foram analisados por meio do SPSS17.0. As análises estatísticas descritivas foram realizadas por meio de cálculos de frequências (absolutas e relativas), bem como por medidas de tendência central e dispersão (média e desvio padrão). Quanto às análises estatísticas inferenciais, o teste qui-quadrado ou exato de Fisher foi utilizado para comparar proporções e o teste t de Student ou ANOVA, um critério para comparar médias, todos com nível de significância de 5\%.

\section{RESULTADO}

Com base nos dados obtidos, as seguintes variáveis foram incluídas no presente estudo: a) dados demográficos: sexo, idade; b) bruxismo: consciência autorrelatada (às vezes e sim); c) ansiedade: tanto para o estado como para o traço, foram categorizados em: não há ansiedade, ansiedade baixa, moderada e grave; d) estresse: sem estresse, fases alerta, resistência e exaustão.

Do total de 2.115 alunos, 714 (33,75\%) universitários, pertencentes a um dos dez cursos de graduação da UFJF-GV, participaram desse estudo. Alunos que não informaram alguma variável da pesquisa não foram contabilizados nas análises estatísticas. A idade média dos participantes foi de 21,5 anos $( \pm 4,53)$ e apenas $8 \%$ participantes não responderam sobre a idade. Quanto a gênero, etnia e estado civil, o maior número de voluntários foi de mulheres e autodeclarados brancos e solteiros, respectivamente.

A maioria dos voluntários autorrelatou que não apresenta o hábito de apertar nem/ou ranger os dentes durante o dia nem durante a noite (Tabela 1).

Tabela 1. Prevalência de bruxismo entre universitários da UFJF/GV

\begin{tabular}{ccc}
\hline Bruxismo & $\mathbf{n}$ & $\mathbf{\%}$ \\
\hline Ausente & 379 & 53.08 \\
Presente & 335 & 46.92 \\
Total & 714 & 100.00 \\
\hline
\end{tabular}

A proporção de alunos sem estresse foi maior, não havendo autorrelatos de estresse na fase de exaustão. Em contrapartida, o nível de ansiedade no momento da aplicação do questionário (ansiedade-estado) foi elevado, de modo que ansiedade grave tanto para estado quanto para traço representou maior porcentagem, 56,30\% e 55,04\%, respectivamente (Tabela 2).

Tabela 2. Prevalência de estresse e ansiedade (estado e traço) entre universitários da UFJF/GV

\begin{tabular}{cccc}
\hline Variável & Categorias & $\mathbf{n}$ & $\mathbf{\%}$ \\
\hline \multirow{2}{*}{ Estresse } & Sem estresse & 665 & 93.14 \\
& Fase alerta & 21 & 2.94 \\
& Fase resistência & 28 & 3.92 \\
Ansiedade - estado & não há ansiedade & 24 & 3.36 \\
& Ansiedade baixa & 112 & 15.69 \\
& Ansiedade moderada & 176 & 24.65 \\
& Ansiedade grave & 402 & 56.30 \\
Ansiedade - traço & Não há ansiedade & 45 & 6.30 \\
& Ansiedade baixa & 117 & 16.39 \\
& Ansiedade moderada & 155 & 21.71 \\
& Ansiedade grave & 393 & 55.04 \\
\end{tabular}


Os resultados obtidos na associação de bruxismo e aspectos demográficos evidenciaram que as proporções foram estatisticamente significantes entre os gêneros, de modo que a proporção do gênero feminino apresentou discreta prevalência de bruxismo, com 50,83\% (Tabela 3).

Tabela 3. Associação entre presença de bruxismo e aspectos demográficos, caracterização do curso e período dos universitários da UFJF/GV

\begin{tabular}{|c|c|c|c|c|c|c|}
\hline \multirow{3}{*}{ Gênero } & \multicolumn{4}{|c|}{ Bruxismo } & \multirow{3}{*}{ Total } & \multirow{3}{*}{ p-valor } \\
\hline & \multicolumn{2}{|c|}{ Ausência } & \multicolumn{2}{|c|}{ Presença } & & \\
\hline & $\mathbf{n}$ & $\%$ & $\mathbf{n}$ & $\%$ & & \\
\hline Feminino & 237 & 49.17 & 245 & 50.83 & 482 & $0.00^{*}$ \\
\hline Masculino & 142 & 61.21 & 90 & 38.79 & 232 & \\
\hline Idade & & & & & & 0.43 \\
\hline Média $\left(\mathrm{dp}^{1}\right)$ & \multicolumn{2}{|c|}{21.4 (4.9) } & \multicolumn{2}{|c|}{$21.6(4.1)$} & $21.5(4.5)$ & \\
\hline Curso & $\mathbf{n}$ & $\%$ & $\mathbf{n}$ & $\%$ & Total & \\
\hline Administração & 30 & 48.39 & 32 & 51.61 & 62 & $0.01^{* * *}$ \\
\hline Ciências Contábeis & 20 & 57.14 & 15 & 42.86 & 35 & \\
\hline Ciências Econômicas & 26 & 70.27 & 11 & 29.73 & 37 & \\
\hline Direito & 77 & 49.68 & 78 & 50.32 & 155 & \\
\hline Educação Física & 18 & 69.23 & 8 & 30.77 & 26 & \\
\hline Farmácia & 35 & 58.33 & 25 & 41.67 & 60 & \\
\hline Fisioterapia & 25 & 43.86 & 32 & 56.14 & 57 & \\
\hline Medicina & 62 & 59.05 & 43 & 40.95 & 105 & \\
\hline Nutrição & 21 & 72.41 & 8 & 27.59 & 29 & \\
\hline Odontologia & 65 & 44.52 & 81 & 55.48 & 146 & \\
\hline Não informado & 0 & 0.00 & 2 & 100.00 & 2 & \\
\hline \multicolumn{7}{|l|}{ Período } \\
\hline 1 & 108 & 65.06 & 58 & 34.94 & 166 & $0.00^{* * *}$ \\
\hline 2 & 67 & 57.76 & 49 & 42.24 & 116 & \\
\hline 3 & 32 & 44.44 & 40 & 55.56 & 72 & \\
\hline 4 & 46 & 58.97 & 32 & 41.03 & 78 & \\
\hline 5 & 8 & 28.57 & 20 & 71.43 & 28 & \\
\hline 6 & 31 & 39.74 & 47 & 60.26 & 78 & \\
\hline 7 & 30 & 50.00 & 30 & 50.00 & 60 & \\
\hline 8 & 20 & 54.05 & 17 & 45.95 & 37 & \\
\hline 9 & 17 & 39.53 & 26 & 60.47 & 43 & \\
\hline Não informado & 20 & 55.56 & 16 & 44.44 & 36 & \\
\hline Total geral & 379 & 53.08 & 335 & 46.92 & 714 & \\
\hline
\end{tabular}

${ }_{1} \mathrm{dp}$ : desvio padrão; ${ }^{*}$ p: valor calculado pelo teste qui-quadrado; ${ }^{* *}$ p: valor calculado pelo teste t de Student; ${ }^{* * *}$ p: valor calculado pelo teste exato de Fisher.

Quanto à caracterização do curso, os resultados evidenciaram que as proporções foram estatisticamente significantes. Entre os dez cursos existentes na instituição, os que apresentaram mais proporção de bruxismo foram Fisioterapia (56,14\%), Odontologia (55,48\%), Administração $(51,61 \%)$ e Direito $(50,32 \%)$. Em contrapartida, os que obtiveram maior índice de ausência de bruxismo foram Nutrição (72,41\%), Ciências Econômicas (70,27\%), Educação Física (69,23\%) e Medicina (59,05\%), respectivamente (Tabela 3).

Quanto ao bruxismo relacionado ao período dos universitários, os resultados evidenciaram que as proporções foram estatisticamente significantes, havendo mais prevalência de bruxismo nos voluntários que cursavam o 5o $(71,43 \%)$, o 9o $(60,47 \%)$ e o 6o período $(60,26 \%)$, respectivamente (Tabela 3). Quanto à idade dos pacientes, não houve significância estatística.

Na associação de bruxismo com estresse, a maioria dos voluntários que relataram não serem bruxistas também não apresentou estresse. Mesmo aqueles que relataram ter bruxismo não apresentaram estresse. Entre os acadêmicos que relataram ausência de bruxismo, 83,33\% não 
estavam ansiosos no momento da aplicação do teste de ansiedade-estado, em contrapartida entre aqueles que relataram ter bruxismo, 52,24\% apresentaram ansiedade grave no momento da aplicação do questionário (Tabela 4).

Tabela 4. Associação de presença de bruxismo com estresse e ansiedade nos universitários da UFJF/GV

\begin{tabular}{|c|c|c|c|c|c|c|}
\hline & \multicolumn{4}{|c|}{ Bruxismo } & \multirow{3}{*}{ Total } & \multirow{3}{*}{ p-valor* } \\
\hline & \multicolumn{2}{|c|}{ Ausência } & \multicolumn{2}{|c|}{ Presença } & & \\
\hline & $\mathbf{n}$ & $\%$ & $\mathbf{n}$ & $\%$ & & \\
\hline \multicolumn{7}{|c|}{ Estresse } \\
\hline Sem estresse & 362 & 54.44 & 303 & 45.56 & 665 & 0.01 \\
\hline Fase alerta & 5 & 23.81 & 16 & 76.19 & 21 & \\
\hline Fase resistência & 12 & 42.86 & 16 & 57.14 & 28 & \\
\hline \multicolumn{7}{|c|}{ Ansiedade - Estado } \\
\hline Não há ansiedade & 20 & 83.33 & 4 & 16.67 & 24 & 0.00 \\
\hline Baixa & 68 & 60.71 & 44 & 39.29 & 112 & \\
\hline Moderada & 99 & 56.25 & 77 & 43.75 & 176 & \\
\hline Grave & 192 & 47.76 & 210 & 52.24 & 402 & \\
\hline \multicolumn{7}{|c|}{ Ansiedade - Traço } \\
\hline Não há ansiedade & 31 & 68.89 & 14 & 31.11 & 45 & $<0.0001$ \\
\hline Baixa & 75 & 64.10 & 42 & 35.90 & 117 & \\
\hline Moderada & 95 & 61.29 & 60 & 38.71 & 155 & \\
\hline Grave & 175 & 44.53 & 218 & 55.47 & 393 & \\
\hline Não informada & 3 & 75.00 & 1 & 25.00 & 4 & \\
\hline
\end{tabular}

* Teste qui-quadrado com nível de significância de 5\%.

Os resultados da associação do bruxismo com a ansiedade-traço mostram que, entre acadêmicos sem bruxismo, 68,89\% não apresentaram ansiedade em seu traço de personalidade. Em compensação, 55,47\% dos pacientes que relataram bruxismo tinham traço de ansiedade grave em sua personalidade (Tabela 4).

\section{DISCUSSÃO}

0 presente estudo mostrou que quase metade dos estudantes universitários autorrelatou ter bruxismo. A palavra bruxismo foi empregada de forma genérica, associando BS e BV. Baseado no novo sistema de classificação diagnóstica elaborado por Lobbezoo et al. $^{2}$, de acordo com as ferramentas empregadas, o bruxismo pode ser categorizado em possível, provável e definitivo: autorrelato (questionários, história oral) para o diagnóstico de possível bruxismo; exame clínico para provável bruxismo; técnicas instrumentais (eletromiografia, polissonografia e estratégias de avaliação em tempo real baseada na abordagem de avaliação ecológica momentânea) para bruxismo definitivo. Dessa forma, de acordo com a abordagem adotada pelo estudo, 46,92\% dos estudantes foram diagnosticados com possível bruxismo, com sutil prevalência no sexo feminino $(50,83 \%)$.

Alguns estudos, com diferentes metodologias aplicadas, avaliaram a prevalência do bruxismo com ampla discrepância dos valores obtidos. Em uma revisão sistemática, Manfredini et al. ${ }^{3}$ apontaram que a prevalência do bruxismo (sem distinção entre BV e BV) entre os estudos selecionados variou de $8 \%$ a $31,4 \%$ e sem relação com sexo. Cavallo et al..$^{13}$ investigaram a prevalência de possíveis BS e BV e constataram prevalência de BV de 37,9\% e de BS de 31,8\%, ambos sem diferença significativa de gênero. Soares et al. ${ }^{14}$ avaliaram a prevalência de provável 
bruxismo em estudantes da Universidade Federal dos Vales Jequitinhonha e Mucuri no Brasil e os resultados mostraram que $31,6 \%$ dos alunos apresentaram bruxismo. No estudo de Huhtela et al. ${ }^{15}, 7,2 \%$ das mulheres e 3,2\% dos homens relataram BS e BV.

Estudos apontaram algumas alterações psicossociais presentes nos universitários ${ }^{10,13,16}$. 0 termo estresse pode ser aplicado para indicar estímulos ou eventos estressantes que geram resposta (ou seja, a entrada anteriormente mencionada) ${ }^{7}$. Estudantes universitários podem sofrer uma quantidade indevida de estresse, oriundo de trabalho acadêmico, situações pessoais e circunstâncias econômicas ${ }^{13}$, além da possibilidade de exercer atividades fora do relógio biológico interno. 0 acúmulo de tarefas e responsabilidades pode gerar estresse e afetar a saúde das pessoas em qualquer idade ${ }^{10}$. Consequências negativas podem refletir em resultados acadêmicos e pessoais, emocionais ou de saúde ${ }^{16}$. Entretanto, nesse estudo, a prevalência de estresse entre universitários foi baixa $(6,84 \%)$, possivelmente justificada pelo momento de coleta dos dados ter sido realizada imediatamente após o recesso escolar.

Para a associação entre bruxismo e estresse emocional, a maioria dos voluntários que autorrelataram ter bruxismo não mencionou estresse. Entretanto, entre aqueles que relataram estresse, a maioria apresentou autorrelato de bruxismo. Estudos anteriores não encontraram associação entre bruxismo definitivo, em especial BS, e autorrelato de estresse ${ }^{17,18}$. Karakoulaki et al. ${ }^{19}$ utilizaram biomarcadores na saliva (cortisol e amilase) para avaliar a relação entre BS definitivo e estresse percebido entre 45 voluntários (30 bruxistas confirmados por registros eletromiográficos - Bitestrip - e 15 não bruxistas) e perceberam que BS estava relacionado a altos níveis de estresse psicológico percebido e cortisol salivar.

Cavallo et al. ${ }^{13}$ investigaram a prevalência de possíveis BS e BV e sua correlação com estresse percebido em um grupo de 278 estudantes de graduação italianos. Uma correlação positiva, com significativas concordância e dependência, entre o escore de estresse e BV esteva presente somente para estudantes do sexo masculino. Os autores afirmaram que os estudantes universitários mostraram altos níveis de BV e estresse e que há correlação entre BV e estresse e diferença de gênero para a presença de estresse. Também afirmaram que a correlação entre estresse e BV é relacionada a gênero, estando presente apenas em estudantes universitários do sexo masculino. Essas divergências de resultados podem ser explicadas pelas diferentes metodologias aplicadas ao diagnóstico do bruxismo e do estresse, além da não distinção do ciclo circadiano do bruxismo.

Soares et al. ${ }^{14}$ também avaliaram fatores associados ao bruxismo em 253 estudantes (106 homens; 147 mulheres) que foram examinados clinicamente e responderam a um questionário. 0 modelo de regressão logística ajustado mostrou que alunos com estresse eram 3,1 vezes mais propensos a ter bruxismo. 0 presente estudo diferiu de estudos prévios ${ }^{20,21}$ que constataram associações entre autorrelato de estresse e bruxismo entre não pacientes. Por outro lado, um trabalho que investigou a quantidade de estresse autorreferido em relação ao bruxismo gravado, eletromiograficamente, durante a noite anterior ao relatório de estresse (estresse antecipado) e na noite seguinte ao relatório (estresse atual), não encontrou associação entre estresse e bruxismo ${ }^{17}$.

Diferindo do estresse, o nível de ansiedade, tanto estado quanto traço, foi elevado, com atenção especial para o nível grave de ambas as condições, acometendo mais da metade dos voluntários no momento da aplicação do questionário e em sua personalidade. Níveis elevados de ansiedade e estresse foram significativamente mais prováveis entre bruxistas autorreferidos frequentes do que entre aqueles que relataram não ter bruxismo leve ${ }^{8}$.

Montero e Gómez-Polo ${ }^{22}$ investigaram a associação entre autorrelatos de fatores psicológicos (nível de ansiedade) e perfis de personalidade (neuroticismo, extroversão, abertura, conveniência e conscientização) com possível bruxismo. Os autores observaram que voluntários que se autodeclararam bruxistas, independentemente de serem do sono ou da vigília, compartilharam um perfil de personalidade comparável e concluíram que o autorrelato de 
bruxismo esteve significativamente associado a vários traços de personalidade (principalmente neuroticismo e extroversão) e ao nível de ansiedade.

Manfredini et al. ${ }^{23}$ sugerem que atividade muscular mastigatória por tempo de sono pode estar mais relacionada à ansiedade-traço do que ao estado de ansiedade ou a outros sintomas psicológicos. Reações ansiosas, especialmente transitórias a eventos estressantes, podem estar relacionadas a autorrelato de bruxismo ${ }^{8}$.

Manfredini et al. ${ }^{9}$ elaboraram uma pesquisa para determinar se existe uma correlação entre características psicológicas e BS. Um total de 36 voluntários saudáveis foi submetido a uma avaliação caseira com um dispositivo portátil combinando gravações eletromiográficas (EMG) e eletrocardiográficas (ECG) para o diagnóstico de BS e aplicação de questionários para avaliar níveis de ansiedade-estado e traço e características e estratégias de enfrentamento. Indivíduos com altos índices psicológicos mostraram ligeira tendência a ter mais episódios de SB, mas as diferenças em relação a indivíduos com níveis baixos e médios de escores psicológicos não foram significativas. Foram encontradas correlações significativas para os escores de ansiedade-estado ( $\mathrm{Phi}=0,456 ; \mathrm{P}=0,006)$, pontuação de ansiedade-traço $(\mathrm{Phi}=0,369 ; \mathrm{P}=0,027)$ e estratégia de enfrentamento de suporte social ( $\mathrm{Phi}=0,387 ; \mathrm{P}=0,020)$.

Quanto à distribuição do autorrelato de bruxismo entre universitários dos diferentes cursos de graduação da instituição, não foram encontradas justificativas que fundamentassem o ocorrido, como carga horária do curso ou estágios extracurriculares. Quanto ao período cursado, as proporções foram maiores nos períodos intermediários e no final. A primeira condição pode ser justificada pela transição entre os ciclos básicos e profissionalizantes, ao passo que a última pode ser decorrente das incertezas das carreiras profissionais pela proximidade da formatura. Sabe-se que o acúmulo de tarefas e responsabilidades cria perturbações psicossociais e pode afetar, adversamente, a saúde ${ }^{16,24}$. Segundo Saddki et al..24, alunos de graduação em saúde enfrentam cargas de trabalho acadêmicas exigentes e devem se concentrar nas tarefas diárias. Assim, são comumente expostos a muitas pressões acadêmicas, que influenciam seu comportamento emocional, devido a reações de adaptação ao estresse ${ }^{14}$. Entretanto, nesse estudo, segundo a metodologia utilizada, não foram encontradas diferenças entre os alunos das áreas de saúde e humanas.

Uma dificuldade encontrada foi, em razão da diversidade de metodologias empregadas, comparar as taxas de prevalência de nossos resultados com estudos anteriores. Assim, essas comparações devem ser consideradas com cautela. Este estudo apresenta algumas limitações, como o emprego de questionário para os diagnósticos, que são práticos para estudos em larga escala, mas, em razão de sua natureza subjetiva, podem gerar resultados supra ou subestimados, além de não distinguir o bruxismo de acordo com o ciclo circadiano, considerando-o entidade única. A única hipótese confirmada desse estudo foi o autorrelato de bruxismo significantemente correlacionado com o estresse e a ansiedade.

\section{CONSIDERAÇÕES FINAIS}

Baseado nos dados obtidos no presente estudo, a maioria dos universitários autorrelatou não ser bruxista, não apresentar estresse e possuir níveis elevados de ansiedade (traço e estado). $\mathrm{Na}$ associação de possível bruxismo com os perfis psicossociais avaliados, a maioria dos voluntários não apresentou estresse, independentemente de terem autorrelatado ou não serem bruxistas. Quanto à ansiedade, entre acadêmicos que relataram ter bruxismo, a maioria apresentou ansiedade (estado e traço) grave no momento da aplicação do questionário. 


\section{REFERÊNCIAS}

1. Manfredini D, Serra-Negra J, Carboncini F, Lobbezoo F. Current concepts of bruxism. Int J Prosthodont. 2017 Sep-Oct;30(5):437-8. http://dx.doi.org/10.11607/ijp.5210. PMid:28806429.

2. Lobbezoo F, Ahlberg J, Glaros AG, Kato T, Koyano K, Lavigne GJ, et al. Bruxism defined and graded: an international consensus. J Oral Rehabil. 2013 Jan;40(1):2-4. http://dx.doi.org/10.1111/joor.12011. PMid:23121262.

3. Manfredini D, Winocur E, Guarda-Nardini L, Paesani D, Lobbezoo F. Epidemiology of bruxism in adults: a systematic review of the literature. J Orofac Pain. 2013;27(2):99-110. http://dx.doi.org/10.11607/jop.921. PMid:23630682.

4. Manfredini D, Lobbezoo F. Relationship between bruxism and temporomandibular disorders: a systematic review of literature from 1998 to 2008. Oral Surg Oral Med Oral Pathol Oral Radiol Endod. 2010 Jun;109(6):e26-50. http://dx.doi.org/10.1016/j.tripleo.2010.02.013. PMid:20451831.

5. Manfredini D, Poggio CE, Lobbezoo F. Is bruxism a risk factor for dental implants? A systematic review of the literature. Clin Implant Dent Relat Res. 2014 Jun;16(3):460-9. http://dx.doi.org/10.1111/cid.12015. PMid:23151302.

6. Klasser GD, Rei N, Lavigne GJ. Sleep bruxism etiology: The evaluation of a changing paradigm. J Can Dent Assoc. 2015;81:f2. PMid:25633110.

7. Manfredini D, Lobbezoo F. Role of psychosocial factors in the etiology of bruxism. J Orofac Pain. 2009;23(2):153-66. PMid:19492540.

8. Ahlberg J, Lobbezoo F, Ahlberg K, Manfredini D, Hublin C, Sinisalo J, et al. Self-reported bruxism mirrors anxiety and stress in adults. Med Oral Patol Oral Cir Bucal. 2013 Jan;118(1):e7-11. http://dx.doi.org/10.4317/medoral.18232. PMid:22926484.

9. Manfredini D, Arreghini A, Lombardo L, Visentin A, Cerea S, Castroflorio T, et al. Assessment of anxiety and coping features in bruxers: a portable electromyographic and electrocardiographic study. J Oral Facial Pain Headache. 2016;30(3):249-54. http://dx.doi.org/10.11607/ofph.1616. PMid:27472528.

10. Serra-Negra JM, Scarpelli AC, Tirsa-Costa D, Guimarães FH, Pordeus IA, Paiva SM. Sleep bruxism, awake bruxism and sleep quality among Brazilian dental students: a cross-sectional study. Braz Dent J. 2014;25(3):241-7. http://dx.doi.org/10.1590/0103-6440201302429. PMid:25252261.

11. Biaggio AMB, Natalício L, Spielberger CD. Desenvolvimento da forma experimental em português do Inventário de Ansiedade Traço-Estado (IDATE) de Spielberger. Arq Bras Psic Apl. 1977;19(3):31-44.

12. Vedolin GM, Lobato VV, Conti PCR, Lauris JRP. The impact of stress and anxiety on the pressure pain threshold of myofascial pain patients. J Oral Rehabil. 2009 May;36(5):313-21. http://dx.doi.org/10.1111/j.1365-2842.2008.01932.x. PMid:19210679.

13. Cavallo P, Carpinelli L, Savarese G. Perceived stress and bruxism in university students. BMC Res Notes. 2016 Dec;9(1):514. http://dx.doi.org/10.1186/s13104-016-2311-0. PMid:28003024.

14. Soares LG, Costa IR, Brum Júnior JS, Cerqueira WSB, Oliveira ES, Douglas de Oliveira DW, et al. Prevalence of bruxism in undergraduate students. Cranio. 2017 Sep;35(5):298-303. http://dx.doi.org/10.1080/08869634.2016.1218671. PMid:27684574.

15. Huhtela OS, Näpänkangas R, Joensuu T, Raustia A, Kunttu K, Sipilä K. Self-reported bruxism and symptoms of temporomandibular disorders in finnish university students. J Oral Facial Pain Headache. 2016;30(4):311-7. http://dx.doi.org/10.11607/ofph.1674. PMid:27792798.

16. Marshall LL, Allison A, Nykamp D, Lanke S. Perceived stress and quality of life among doctor of pharmacy students. Am J Pharm Educ. 2008 Dec;72(6):137. http://dx.doi.org/10.5688/aj7206137. PMid:19325957.

17. Pierce CJ, Chrisman K, Bennett ME, Close JM. Stress, anticipatory stress, and psychologic measures related to sleep bruxism. J Orofac Pain. 1995;9(1):51-6. PMid:7581205. 
18. Nakata A, Takahashi M, Ikeda T, Hojou M, Araki S. Perceived psychosocial job stress and sleep bruxism among male and female workers. Community Dent Oral Epidemiol. 2008 Jun;36(3):201-9. http://dx.doi.org/10.1111/j.1600-0528.2007.00388.x. PMid:18474052.

19. Karakoulaki S, Tortopidis D, Andreadis D, Koidis P. Relationship between sleep bruxism and stress determined by saliva biomarkers. Int J Prosthodont. 2015 Sep-Oct;28(5):467-74. http://dx.doi.org/10.11607/ijp.4296. PMid:26340005.

20. Ahlberg J, Rantala M, Savolainen A, Suvinen T, Nissinen M, Sarna S, et al. Reported bruxism and stress experience. Community Dent Oral Epidemiol. 2002 Dec;30(6):405-8. http://dx.doi.org/10.1034/j.1600-0528.2002.00007.x. PMid:12453110.

21. Ahlberg K, Ahlberg J, Könönen M, Partinen M, Lindholm H, Savolainen A. Reported bruxism and stress experience in media personnel with or without irregular shift work. Acta Odontol Scand. 2003 Oct;61(5):315-8. http://dx.doi.org/10.1080/00016350310006753. PMid:14763785.

22. Montero J, Gómez-Polo C. Personality traits and dental anxiety in self-reported bruxism. A crosssectional study. J Dent. 2017 Oct;65:45-50. http://dx.doi.org/10.1016/j.jdent.2017.07.002. PMid:28684309.

23. Manfredini D, Fabbri A, Peretta R, Guarda-Nardini L, Lobbezoo F. Influence of psychological symptoms on home-recorded sleep-time masticatory muscles activity in healthy subjects. J Oral Rehabil. 2011 Dec;38(12):902-11. http://dx.doi.org/10.1111/j.1365-2842.2011.02226.x. PMid:21569074.

24. Saddki N, Sukerman N, Mohamad D. Association between emotional intelligence and perceived stress in undergraduate dental students. Malays J Med Sci. 2017 Mar;24(1):59-68. http://dx.doi.org/10.21315/mjms2017.24.1.7. PMid:28381929.

\section{CONFLITOS DE INTERESSE}

Os autores declaram não haver conflitos de interesse.

\section{*AUTOR PARA CORRESPONDÊNCIA}

Ana Paula Varela Brown Martins, Universidade Federal de Juiz de Fora, Departamento de Odontologia, Campus Governador Valadares, Avenida Dr. Raimundo Monteiro Rezende, 330, Centro, 35010-177 Governador Valadares - MG, Brasil, Tel: +55(33) 3301-1000 (1580); anapaula.martins@ufjf.edu.br

Recebido: Janeiro 18, 2021

Aprovado: Março 2, 2021 